\title{
Effective Collaborative Work Utilising Videoconferencing
}

Northern Ireland - Toyama Videoconference Link Project

\author{
Yoshinori Naruse, David Farrell and Jun-ichi Yamanishi \\ Toyama National College of Maritime Technology, 1-2 Ebie-Neriya Shinminato, Toyama 933- \\ 0293, Japan \\ naruse@toyama-cmt.ac.jp \\ Ballyclare Secondary Schoo, Doagh Road, Newtownabbey, N Ireland BT39 9BG, U.K \\ Faculty of Education, Toyama University, 3190 Gofuku, Toyama 930-8555, Japan
}

Keywords: collaborative learning, electronic communication, motivation, videoconferencing

\begin{abstract}
Since September 1991 the Japanese Studies Project has linked twenty schools in Northern Ireland with schools in Japan. The videoconference links proved to be interesting and extremely motivating for the students involved, but it was recognised that a more structured approach would be required in order for all parties to obtain maximum benefit from the links. After considerable discussion it was decided that we should create a collaborative assignment which would require students in both countries to utilise as many skills as they could, in order to communicate effectively and to learn something about each other's culture.
\end{abstract}

\section{INTRODUCTION}

At present most schools in Northern Ireland and Japan are connected to the Internet and with the introduction of the cross-curricular studies module in Japan there is the necessity to have effective examples of international links. In both countries it has been decided to incorporate the use of ICT into 
the subjects being taught rather than as an appendix to the educational system. In other words we seek to educate using ICT rather than educate about ICT (Lynch et al. 2000).

Since September 1991 the Japanese Studies Project has linked twenty schools in Northern Ireland with schools in Japan. As a result of the Project, students in schools in Northern Ireland have been introduced to the Japanese language, electronic mail links with partners in Toyama and access to the World Wide Web. Japanese students have also had the opportunity to develop language skills and have been introduced to developing information communication infrastructure. Early links were heavily dependant upon the use of email to exchange information about themselves, their schools and their towns. While this was very effective in the early 1990's it became increasingly obvious that as the technology advanced the opportunity was being created for collaborative work between the students (Naruse 1993).

As a result of considerable discussion between us, by email and reciprocal visits to Japan and Northern Ireland, it was decided the time had come to expand the opportunity for by utilising videoconferencing.

Early experiments in videoconferencing between Toyama and Northern Ireland in 1996-1997 had clearly shown that it was a viable means of communication between students in Japan and Northern Ireland but the major obstacle was the connection cost. Videoconferencing requires the use of two ISDN lines to Japan therefore each call is double the normal rate to Japan.

In the spring of 1998 we decided to pursue funding for this type of link. In 1998 we were provided the funds to allow videoconference links between Toyama University and a school in Northern Ireland to commence. These initial links took place once or twice a month. Another school was in Japan was provided with funds to allow videoconference links which would commence in September 1999.

The video-conference links in the academic year 1998-1999 proved to be interesting and extremely motivating for the students involved, but it was recognised at the end of the academic year, that a more structured approach would be required in order for all parties to obtain maximum benefit from the links (Figure 1). 


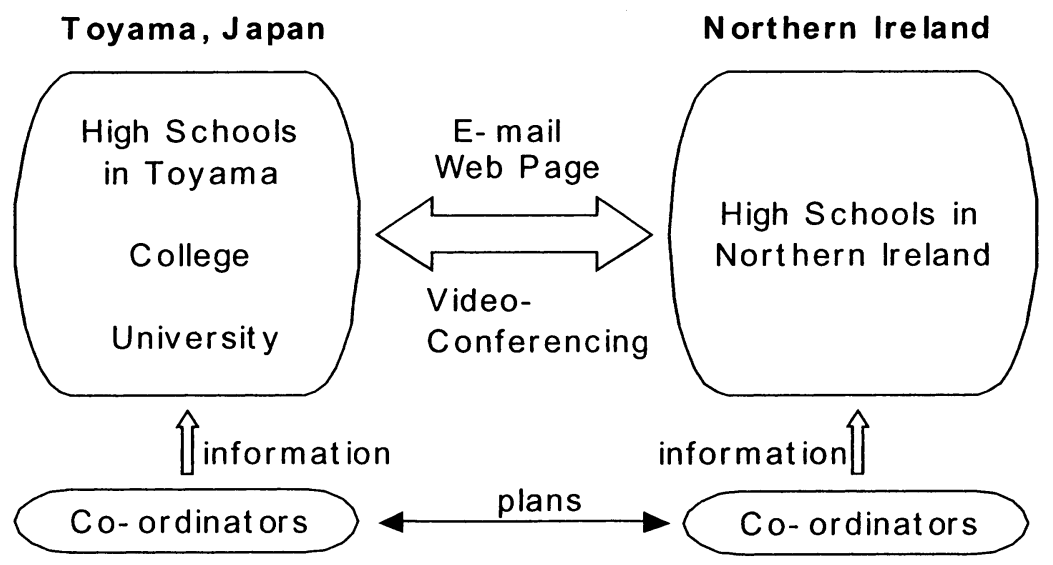

Figure 1. Northern Ireland - Toyama Videoconference Link Project

\section{RECENT DEVELOPMENTS}

In August 1999, we agreed to establish a more structured project between schools in Toyama and schools in Northern Ireland. After considerable discussion it was decided that we should create a collaborative assignment that would require students in both countries to utilise as many skills as they could, in order to communicate effectively and to learn something about each other's culture.

Many educational projects using computer mediated communication have been attempted, linking classes together to work on pre-arranged collaborative work. Some projects have adopted the joint problem solving style where students could share ideas and experiences about issues presented to them (Robinson 1998).

Such a collaborative piece of work which would allow students an opportunity to examine the culture and language of their respective partner countries was required and also provide them with the opportunity to develop their ICT skills as required by the curriculum in each country.

In September 1999 Japanese co-ordinators visited Northern Ireland for their research. During this visit, time was taken out to arrange a meeting with three local schools and an officer of the North Eastern Education and Library Board (NEELB) to discuss an assignment which could last from October 1999 to January 2000 and which could be incorporated into their Japanese Studies classes. During the meeting the details were presented to the teachers 
from the three other schools and after minor modifications it was accepted by all and the structure and timing of the exercise was agreed.

The Project co-ordinators have recognised the importance of using the synchronous communications in collaborative studies between students. The synchronous communications, such as face to face communication, have immediacy in exchanging ideas (Ingram et al. 2000). As for situations when students cannot communicate face-to-face, equivalent communications have been achieved on the Internet and using video conferencing equipment. But because of the problem of the line fee and the time difference (8-9 hours) students are not allowed to use video conferencing equipment with any degree of frequency.

This type of communication was held for the following purpose:

- To establish friendship between students

- To motivate the student to take part in the study of each other's community

- To make students report back on the outcome of their studies with each other.

\section{ASSIGNMENT DETAILS}

\subsection{Objectives}

To raise awareness of the language, geography and culture of each other's country.

To raise awareness in the use of information technology, including:

- Electronic mail

- Internet Access

- Word Processing

- Spreadsheets

- Web Page Creation

- Video Conferencing.

To improve communication skills.

\subsection{Task}

Using a budget of $\$ 1500$ students are to plan a visit to each other's country. Using this budget they are to book flights, find accommodation, visit places of interest and travel in Japan or the United Kingdom. 


\subsection{Rules}

1. Students from Northern Ireland must spend at least three days in Toyama and students from Japan must spend at least three days in Northern Ireland

2. Students must only use the Internet, email and video-conferencing to get information about their respective countries

3. Students must not write to agencies, travel agents or embassies to gain information

4. Students should keep all data as evidence to be used when they are compiling their final report. The task must be completed by 31 December 1999.

\subsection{Procedure}

Students should use the Internet:

- to find out information about the geography of the country they intend to visit

- to investigate the costs of flights to that country

- to create an itinerary for the main areas of interest they wish to visit

- to find out the details of the cost of transportation around the country

- to find details of hotels/hostels etc.

- to work out a daily budget for their stay in each country

- to decide what time of the year they wish to visit the country.

They may wish to engage in sporting activities or participate in festivals.

\subsection{Guidelines}

Each school can enter one team. All participating schools can access the mailing list set up to answer queries about Japan or Northern Ireland. Students can access the mailing list as often as they wish to ask questions or to seek help with their proposed trip. Their mailing list is their 'tour guide' and we will promise to answer all email enquiries within 48 hours.

Students can also have a personal link with a student in their partner school. This information will not be regulated and can be on a one to one basis. 


\subsection{Timescale}

PHASE 1

October 1999 NI students email Japan asking questions about a potential trip to Japan. Emails must be brief and contain no more than three questions.

PHASE 2

November 1999 Japanese schools email NI asking questions about their trip

PHASE 3

December 31 Schools can communicate with each other without restriction, asking questions and making final plans.

Final presentation forwarded to Toyama University to be placed on the home-page

\subsection{Video conference}

Every three weeks a videoconference lasting 20 minutes was to be held in each school. This would allow students an opportunity to discuss each other's culture and should have been active and creative. Role-play was recommended. For example students in Northern Ireland may be shown how to enter a Japanese house and what they should say or students in Japan may wish to know how to ask directions from a person on the street. This can be demonstrated by videoconference. The videoconference could also discuss areas of possible cultural interest or examples of food.

\subsection{Final report}

Students must create a web page detailing their trip. All costs must be clearly given. It must be a detailed account of how they will spend their 1500 or the equivalent in Japanese yen. Key places of interest should be identified and students should simply state why they would like to visit the location they have chosen.

\section{EVALUATION}

To date, the assignment has worked very well between Ballyclare Secondary and Toyama Maritime College and has made a major contribution to Japanese Studies within the school. The other three schools involved had a mixed experience. One school faced major technical problems that prevented 
effective videoconferencing to take place. Due to the fact that the other schools had no previous experience of videoconferencing with Japan, it was no surprise that teething problems were identified. These problems are part of the learning experience and the lessons will be incorporated into next year's plans.

It was intended to complete the assignment by December 1999 but this date was completely unrealistic. The deadline was subsequently extended to the end of April.

It was hoped that schools would be able to submit a completed home page by this date. However, not all schools could manage to submit the final page as planned, however all are in agreement that the exercise has been very beneficial in promoting each other's culture and lifestyle and developing ICT skills. The students have had regular email contact with their partners, access to web pages created by their partners in Toyama, a bulletin board/discussion area and videoconferencing.

For all schools involved it has been a learning experience which will be built upon in the next academic year, but an experience which has effectively introduced participating students to each others culture. Negotiations have already commenced between schools in Northern Ireland, Toyama University and Toyama Maritime College as to how the project could be improved during the next academic year and how we could consolidate the work which has been commenced.

\section{CONCLUSIONS}

To date the link has been successful but what are the lessons to be learnt?

- In order to have effective communication and to establish collaborative work between schools it is essential for close liaison between the teachers and co-ordinators of the Project before the links commence.

- Financial implications must be considered and agreed before links commence.

- The collaborative work to be undertaken must be clearly understood by all parties involved.

- The teachers involved must have the basic ICT skills however as Cifuentes and Murphy (2000) report a result of the links which have been established is that teachers who have become involved in collaborative work have also sought to master their ICT skills.

- Student motivation increases with the use of video-conferencing in collaborative work. Students wish to learn more of their partner's language so that simple communication can be established before the collaborative work commences. 


\section{REFERENCES}

Cifuentes, L. and Murphy, K. L. (2000) Promoting multicultural understanding and positive self-concept through a distance leaming community: Cultural connections. Educational Technology Research and Development, 48, 1, pp. 69-83.

Ingram, A. L., Hathorn, L. G. and Evans, H. (2000) Beyond chat on the internet. Computer \& Education, 35, pp. 21-35.

Lynch, W., Hobbs, B. and Hollanders, H. (2000) Dancing on quicksand, The role of the ICT co-ordinator in the primary school, Research in Education, 62, pp. 32-40.

Naruse, Y. (1993) Educational projects making use of international tele-communication. Research Studies of Toyama National College of Maritime Technology, 26, pp. 95-102.

Robinson, D. (1998) Student portfolios in mathematics. The Mathematics Teacher, 91, 4, pp. 318-325.

\section{BIOGRAPHY}

Yoshinori Naruse is an Associate Professor of Mathematics. His current research interest is the assessment utilising ICT. He has researched on CAI and collaborative studies using the Internet and videoconferencing. He is leading schools in the Province of Toyama which are linked with Northern Ireland and working with teachers of k-12 to promote students' motivation for their studies with ICT.

David Farrell is Vice Principal of Ballyclare Secondary School in Northern Ireland and Consultant to the Education and Library Boards in Northern Ireland for Japanese Studies. During the 1991 Japan Festival in the United Kingdom, he established one of the earliest ICT links between schools in the United Kingdom and Japan. This link is still going and recently it has incorporated the use of video conferencing with schools in Japan.

Jun-ichi Yamanishi is a Professor of the Department of Computer and Instructional Sciences in Toyama University. He has been researching ICT and Integrated Studies using the Internet in k-12 in Japan and recently developed a Web-based instruction for lifelong education. He is working for on an educational link between US and Toyama in addition to a link with Northern Ireland. 